\title{
Influence of Dielectric Loading on the Fidelity Factor of an Ultra Wideband Monopole Antenna
}

\author{
Mohsen Koohestani ${ }^{1,2}$, Nuno Pires ${ }^{1,2}$, Anja K. Skrivervik ${ }^{2}$, Antonio A. Moreira ${ }^{1}$ \\ ${ }^{I}$ Instituto de Telecomunicações, Instituto Superior Técnico, Universidade Técnica de Lisboa \\ Av. Rovisco Pais 1, 1049-001 Lisboa, Portugal \\ ${ }^{2}$ Laboratoire d'Electromagnétisme et d'Acoustique, École Polytechnique Fédérale de Lausanne \\ STI-IEL, Station 11, CH-1015 Lausanne, Switzerland
}

koohestani.mohsen@ist.utl.pt, nuno.pires@ist.utl.pt, anja.skrivervik@epfl.ch, antonio.moreira@lx.it.pt

\begin{abstract}
This paper reports a study of the influence on the fidelity factor due to loading an Ultra Wideband monopole with a pair of dielectric substrate pads. The fidelity factor was calculated, with and without loading, using the simulated transmitted and received pulses in three points in the azimuth plane. Results show that this dielectric loading technique does not cause significant pulse distortion. The fidelity factor is affected only by an average of $2.8 \%$ relative to the unloaded antennas in all situations considered. Two versions of the same antenna design were studied: coplanar- and microstrip-fed. It was found that the influence of feeding on the fidelity factor is also minimal.
\end{abstract}

Keywords-UWB monopole antenna; dielectric loading; fidelity factor; microstrip-fed; $C P W-f e d$

\section{INTRODUCTION}

The demand for Ultra-wide Band (UWB) antennas has required significant development efforts to achieve small designs for a variety of applications such as consumer devices, wireless body area network, radar, biomedical imaging, and localization [1-6].

The effects on UWB monopoles of a dielectric loading sandwich technique, consisting of gluing two commercial substrate pads on both sides of an antenna, have been previously studied and presented [1-2]. It was found that this technique increases the antenna electrical size and improves matching resilience without significant efficiency decrease. Other possible antenna performance trade-offs need to be evaluated, therefore, this paper aims at studying the impact on the fidelity factor of this technique.

Effects on the time performance of dielectric loading are, to the best of the authors' knowledge, still unreported. The most used parameter to assess time performance is the antenna fidelity factor [7-8]. In our study, the fidelity factor was calculated with and without the sandwich padding. The same UWB monopole base design was considered with two different common feeding structures: microstrip- (MS-fed) and coplanar-fed (CPW-fed) in order to also evaluate the influence of the feeding structure on fidelity.

\section{ANTENNA DESIGN AND STRUCTURE}

Two identical printed UWB antennas with different feeding structures were designed and simulated. Their configuration and

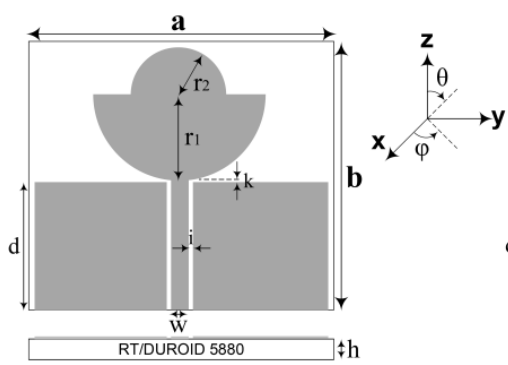

(a)

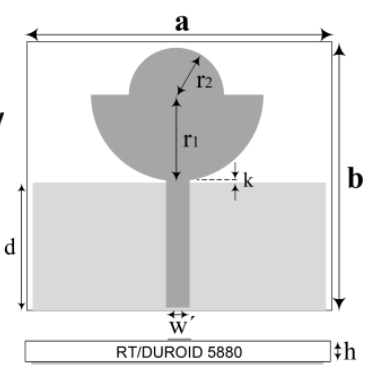

(b)
Figure 1. Printed UWB monopole antennas: (a) CPW-fed, (b) MS-fed

TABLE I. OPTIMAL PARAMETER VALUES OF BOTH STRUCTURES IN MM

\begin{tabular}{c|c|c|c|c|c|c|c|c|c}
$\boldsymbol{a}$ & $\boldsymbol{b}$ & $\boldsymbol{r}_{1}$ & $\boldsymbol{r}_{2}$ & $\boldsymbol{d}$ & $\boldsymbol{w}$ & $\boldsymbol{k}$ & $\boldsymbol{i}$ & $\boldsymbol{h}$ & $\boldsymbol{w}^{\prime}$ \\
\hline 44 & 38 & 11 & 7.5 & 18 & 3.2 & 0.2 & 0.15 & 1.57 & 4.9
\end{tabular}

dimensional parameters are shown in Fig. 1. In both versions, the radiator patch comprises two semicircles with different radii. The backside of the $\mathrm{CPW}$-fed antenna substrate is devoid of any metallization while the backside of the MS-fed antenna supports a finite ground plane. The two designs differ in the feeding line width ( $w$ for CPW and $w^{\prime}$ for MS) that was calculated to have an input impedance of $50 \Omega$ in both structures. The used substrate was RT/Duroid ${ }^{\mathrm{TM}} 5880$ with thickness of $1.57 \mathrm{~mm}$, relative permittivity of 2.2 and loss tangent of 0.0009 . To achieve the desired antenna performance, e.g. wider impedance characteristic, the dimensions of the proposed antenna must be optimized [3]. The final dimensions are presented in Table 1. Among the dimensional parameters shown in Table I, the performance of both antennas is mainly affected by the feed gap $(k)$, the radiator shape $\left(r_{1}, r_{2}\right)$ and the ground plane size.

The dielectric loading sandwich technique was applied to both antennas adequately choosing pad size and permittivity. Two identical rectangular slices of de-metalized commercial substrate $\left(\mathrm{RO}_{3003}{ }^{\mathrm{TM}}\right)$ with a permittivity of 3.0 , loss tangent of 0.001 and thickness of $1.52 \mathrm{~mm}$ were padded on each side of the two structures, as seen in Fig. 2 for the CPW-fed antenna prototype. The width and length of the dielectric pads are 19 and $22 \mathrm{~mm}$, respectively. It should be noted that larger loading pads cause a higher loading effect, i.e., the antenna has enhanced

This work was supported by the Portuguese Fundação para a Ciência e a Tecnologia (FCT) project PEst-OE/EEI/LA0008/2011. The work of Nuno Pires is supported by the FCT grant SFRH/BD/51066/2010 and Mohsen Koohestani by the FCT grant SFRH/BD/51445/2011. 


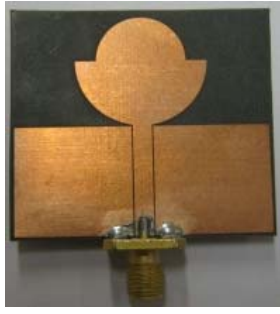

(a)

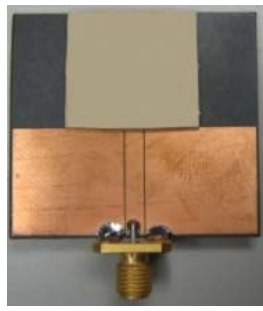

(b)
Figure 2. CPW-fed built prototype: (a) without loading, (b) with loading

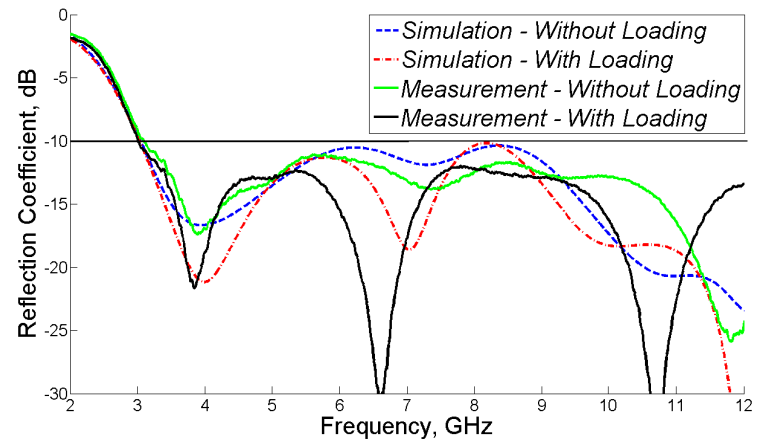

(a)

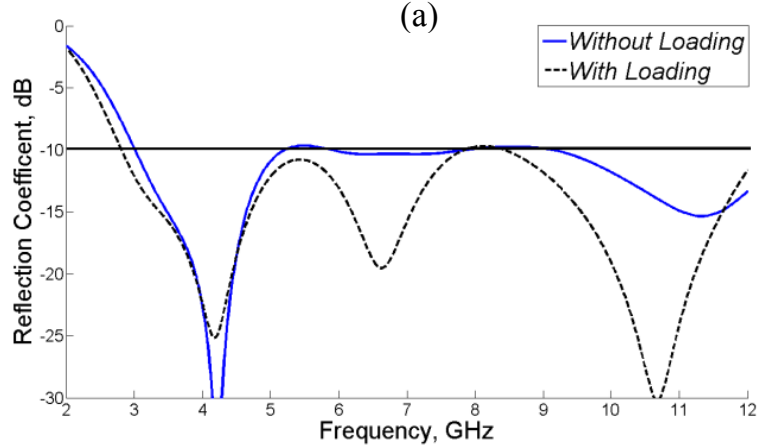

(b)

Figure 3. Simulated reflection coefficient results of antenna:

(a) CPW-fed, (b) MS-fed

matching level, although lower radiation efficiency is expected. The loaded antennas dimensions were not further adjusted taking the sandwich into account in order to have a fair performance comparison in the time domain between the loaded and unloaded versions.

\section{RESULTS AND DISCUSSION}

Results for both frequency and time domain are reported in this section. Simulations have been carried out using the commercial software package CST Microwave Studio ${ }^{\mathrm{TM}} 2012$.

\section{A. Frequency Domain}

Fig. 3 illustrates the reflection coefficient of the designed antennas with and without loading. Experimental results are also presented for the CPW-fed prototype (Fig. 2) that was built and measured to confirm the validity of the simulation model with and without loading.

Both unloaded antennas have an input impedance bandwidth extending in the range 3 to $12 \mathrm{GHz}$, fulfill the requirement of VSWR $<2$ (equivalent to $\mathrm{S}_{11}<-9.5 \mathrm{~dB}$ ), and cover more than the FCC-defined frequency band.

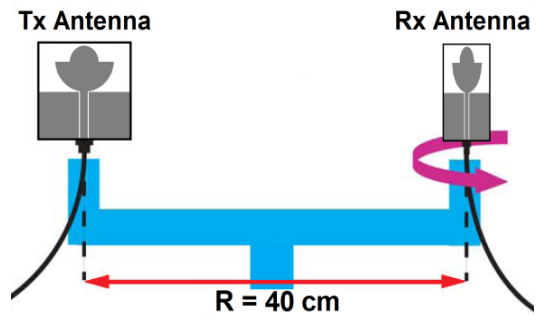

Figure 4. Simulation setup

For the loaded antennas the operating bandwidth is mainly affected in the lower frequencies.

\section{B. Time Domain}

The time-domain simulation setup is depicted in Fig. 4. It uses two identical antennas for transmitting (Tx) and receiving $(\mathrm{Rx})$. The Tx antenna is fixed and is facing the $\operatorname{Rx}$ antenna $(\varphi=$ $\left.0^{\circ}, \theta=90^{\circ}\right)$. The $\mathrm{Rx}$ antenna rotates in the azimuth plane $(\varphi=$ $0^{\circ}, 90^{\circ}$, and $180^{\circ}, \theta=90^{\circ}$ ). The chosen distance between the antennas is $40 \mathrm{~cm}$, which is four times the wavelength at the lower operating frequency $(3 \mathrm{GHz})$, large enough to validate the far-field approximation.

A Gaussian modulated sine pulse (CST default excitation) was used as the source pulse with spectrum corresponding to the $3.1 \mathrm{GHz}-10.6 \mathrm{GHz}$ frequency range. Figs. 5 and 6 illustrate the impulse responses for both antennas with and without loading. The results show good transient performance of both antennas at different orientations. In the plots, the received signals $(\mathrm{Rx})$ with and without loading are similar; hence, the technique does not impose significant pulse distortion. a well-known parameter, the fidelity factor [7], has been used to quantify the pulse distortion level. It basically compares the shapes of a source pulse, $\mathrm{S}_{\mathrm{t}}(\mathrm{t})$, and a received pulse, $\mathrm{S}_{\mathrm{r}}(\mathrm{t})$, and it has been defined by the Equation below (see [9], for instance).

$$
\max _{\tau} \int_{-\infty}^{+\infty} \frac{S_{t}(t)}{\left|S_{t}(t)\right|^{2}} \cdot \frac{S_{r}(t-\tau)}{\left|S_{r}(t)\right|^{2}} d t
$$

The fidelity is a parameter that estimates the maximum correlation coefficient of the two signals by varying the time delay $\tau$. In fact, it reflects the similarity between the transmitted and received pulses. The fidelity factor of the considered structures was calculated by using the Rx and Tx pulses of Fig. 5 and 6 and the results are shown in Table II. For the studied antennas the fidelity factor is minimally affected by the feeding structure with an average deviation of $1.8 \%$ of the CPW-fed antenna to the MS-fed antenna for every orientation. The simulation results show that dielectric loading does not significantly impact fidelity factor of both antennas with a degradation of only $2.8 \%$ on average and relative to the unloaded antennas in all situations considered. The worst case, with a $10 \%$ decrease in the parameter, occurs at $\varphi=90^{\circ}$ for both structures. This is due to the different radiation patterns of the loaded vs. unloaded antennas. Unloaded antennas have higher directivity in $\varphi=90^{\circ}$ and loading causes a decrease in the same direction. The fidelity factor in $\varphi=90^{\circ}$ is therefore higher for the unloaded antennas and lower when loading is applied. It should be noted that both structures have lower gain in the $9 \mathrm{GHz}$ region leading to an overall reduction of the 


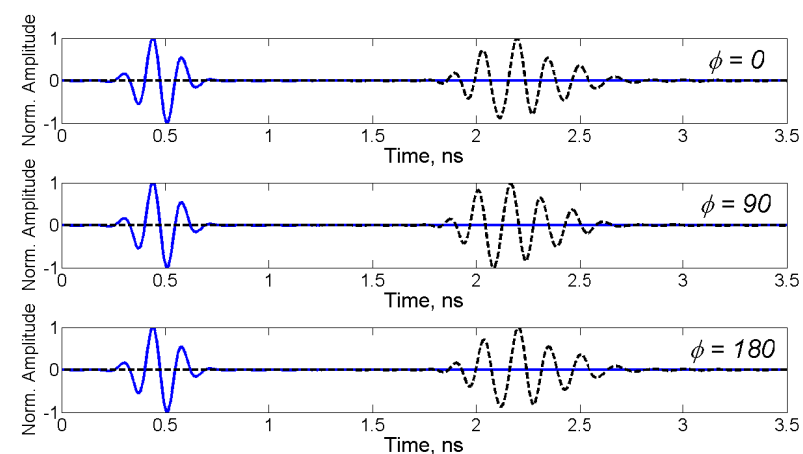

(a)

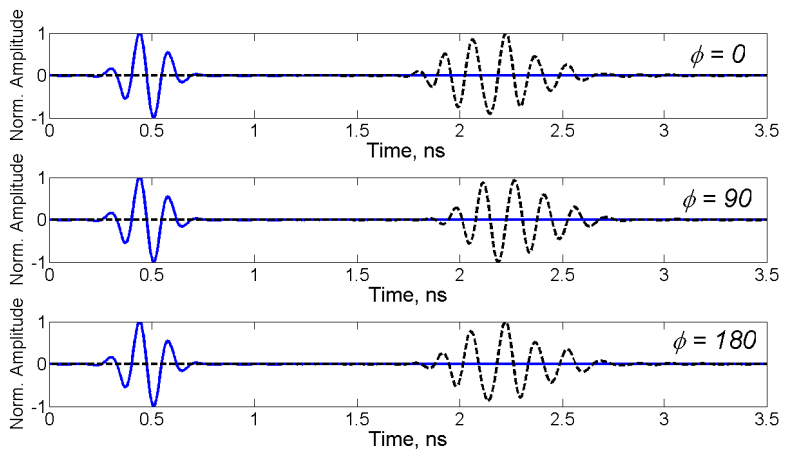

(b)

Figure 5. Tx and Rx Signal of the Antenna without Loading: (a) CPW-fed, (b) MS-fed

fidelity factor values. The parameter has an average of about 0.84 in this study, which is higher than the commonly accepted minimum of $0.5[8]$.

\section{CONCLUSION}

A study of the influence of dielectric loading on the fidelity factor of an UWB printed monopole antenna has been presented. The following observations can be made: proper loading does not impose significant distortion on the transmitted pulses which leads to an average of $2.8 \%$ deviation in fidelity factor from the unloaded antennas; it has been confirmed that adequate loading does not significantly detune the antennas.

This study also aimed at evaluating the importance of feeding structure. To this goal, two versions of the same UWB printed monopole antenna have been studied: CPW- and MSfed. Antenna input matching has been simulated and measured for one of the designs to confirm the model accuracy. It was found that the antenna feeding structure has minimal impact on the fidelity factor with a $1.8 \%$ average deviation from $\mathrm{CPW}$ - to MS-fed.

\section{REFERENCES}

[1] Moreira, A.A.; Pires, N.; Serro, N.; Santos, R., "Ultra wideband dielectric sandwich loaded antennas," 3rd European Conference on Antennas and Propagation, Berlin,Germany, Mar. 2009.

[2] N. Pires, M. Letizia, S. Boyes, Y. Lu, Y. Huang, A. K. Skrivervik, and A. Moreira, "Improved design of an Ultra Wideband Universal Serial Bus Device mounted antenna based on comparative radiation efficiency measurements," 5th European Conf. on Antennas and Propag. Rome, Italy, Apr. 2011.

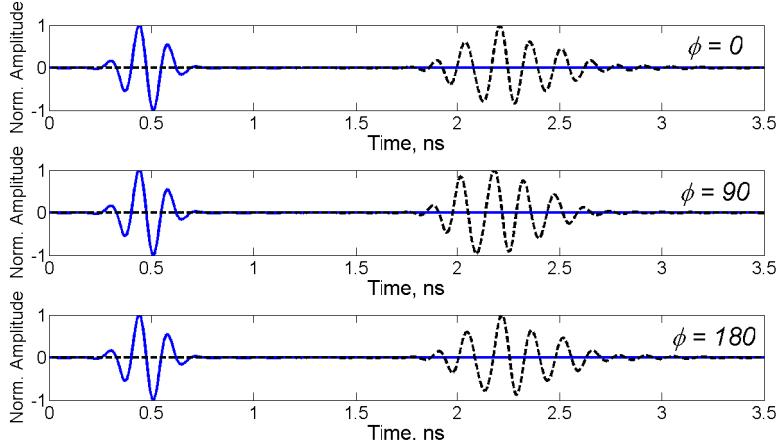

(a)

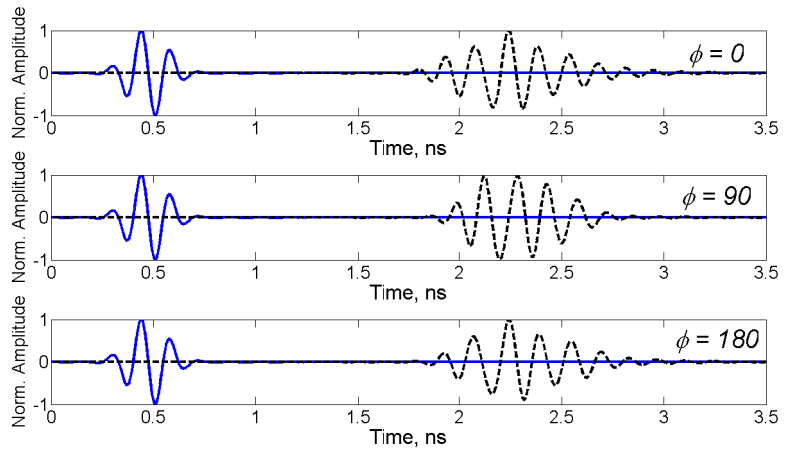

(b)

Figure 6. Tx and Rx Signal of the Antenna with Loading:

(a) CPW-fed, (b) MS-fed

TABLE II. FIDELITY FACTOR

\begin{tabular}{|c|c|c|c|}
\hline \multirow{2}{*}{ Antenna Type } & \multirow{2}{*}{ Phi (deg) } & \multicolumn{2}{|c|}{ Fidelity Factor } \\
\cline { 2 - 4 } & & Without Loading & With Loading \\
\hline \multirow{3}{*}{ CPW-fed } & $\boldsymbol{\varphi}=\mathbf{0}$ & 0.83 & 0.83 \\
\cline { 2 - 4 } & $\boldsymbol{\varphi}=\mathbf{9 0}$ & 0.86 & 0.81 \\
\cline { 2 - 4 } & $\boldsymbol{\varphi}=\mathbf{1 8 0}$ & 0.83 & 0.83 \\
\hline \multirow{3}{*}{ MS-fed } & $\boldsymbol{\varphi}=\mathbf{0}$ & 0.83 & 0.82 \\
\cline { 2 - 4 } & $\boldsymbol{\varphi}=\mathbf{9 0}$ & 0.88 & 0.79 \\
\cline { 2 - 4 } & $\boldsymbol{\varphi}=\mathbf{1 8 0}$ & 0.81 & 0.81 \\
\hline
\end{tabular}

3] M. Koohestani, N. Pires, A. K. Skrivervik, and A. A. Moreira, "Influence of the human body on a new coplanar-fed ultra-wideband antenna," 6th European Conf. on Antennas and Propag., Prague, Czech Republic, Apr. 2012.

[4] N. Chahat, M. Zhadobov, R. Sauleau, and K. Ito, "A compact UWB antenna for on-body applications," IEEE Trans. on Antennas and Propag., vol. 59, no. 4, April 2011.

[5] Allen, B., Dohler, M., Okon, E.E., Malik,W.Q., Brown, A.K., Edwards, D.J.: 'Ultra-wideband antennas and propagation for communications radar and imaging' (John Wiley \& Sons, 2007).

[6] Sanming Hu; Yuan Zhou; Choi Look Law; Wenbin Dou; , "Study of a Uniplanar Monopole Antenna for Passive Chipless UWB-RFID Localization System," IEEE Trans. on Antennas and Propag., vol.58, no.2, pp.271-278, Feb. 2010

[7] E. Pancera, T. Zwick, and W.Wiesbeck, "Spherical Fidelity Patterns of UWB Antennas" IEEE Trans. on Antennas and Propag., vol. 59, 2011.

[8] Quintero, G.; Zurcher, J.-F.; Skrivervik, A. K. , "System Fidelity Factor: A New Method for Comparing UWB Antennas," IEEE Trans. on Antennas and Propag., vol.59, no.7, July 2011.

[9] O. E. Allen, D. A. Hill, and A. R. Ondrejka, "Time-domain antenna characterizations," IEEE Trans. Electromagn. Compat., vol. 35, Aug. 1993. 